\title{
Protective effect of propofol via the regulation of ovarian granulosa cell proliferation and apoptosis
}

\author{
RONG DING* ${ }^{*}$, WENYUE KANG ${ }^{*}$, DUOZHI WU and LIN WANG \\ Department of Anesthesiology, Hainan General Hospital (Hainan Hospital Affiliated to \\ Hainan Medical University), Haikou, Hainan 570311, P.R. China
}

Received February 24, 2020; Accepted March 15, 2021

DOI: $10.3892 /$ etm.2021.10420

\begin{abstract}
Propofol is an anesthetic frequently used in surgery. Accumulating evidence suggests that propofol exhibits an effect on cell viability, apoptosis and invasion in several types of cancer cells. MicroRNAs (miRNAs) have been reported to play pivotal roles in the development of polycystic ovary syndrome (PCOS). However, the diagnostic applications of miR-451a in PCOS remain unknown. The present study aimed to elucidate the effects of propofol on ovarian granulosa cell proliferation and apoptosis and illustrate the specific mechanisms associated with this process. Human ovarian granulosa cell-like KGN cells, which were used as a representative of granulosa cells in the present study, were treated with different concentrations $(0,1,5$ and $10 \mu \mathrm{g} / \mathrm{ml})$ of propofol for $48 \mathrm{~h}$ and cell proliferation and apoptosis were assessed using MTT and flow cytometry assays, respectively. Propofol treatment resulted in significant inhibition of cell viability and induction of apoptosis in KGN cells, which was accompanied with increased cleaved caspase 3 and suppressed pro-caspase 3 expression levels. Furthermore, propofol reduced Wnt $3 \mathrm{a}$ and $\beta$-catenin protein and mRNA expression levels. miR-451a expression in KGN cells was evaluated by reverse transcription-quantitative PCR (RT-qPCR). miR-451a expression was upregulated in propofol-stimulated KGN cells. The data further demonstrated that miR-451a mimics suppressed cell proliferation and increased apoptosis of KGN cells compared with cells transfected with control mimics. Furthermore, the association between miR-451a and propofol was investigated. Rescue experiments were performed to investigate the anti-proliferative mechanism of propofol in
\end{abstract}

Correspondence to: Mrs. Wenyue Kang, Department of Anesthesiology, Hainan General Hospital (Hainan Hospital Affiliated to Hainan Medical University), 19 Xiuhua Road, Haikou, Hainan 570311, P.R. China

E-mail:kk2222345@163.com

*Contributed equally

Key words: propofol, polycystic ovary syndrome, ovarian granulosa cells ovarian granulosa cells. KGN cells were transfected with miR-451a inhibitor or inhibitor control sequences for $6 \mathrm{~h}$ and treated with $10 \mu \mathrm{g} / \mathrm{ml}$ propofol for an additional $48 \mathrm{~h}$. The results from the MTT, RT-qPCR and western blot assays indicated that $10 \mu \mathrm{g} / \mathrm{ml}$ propofol inhibited cell viability, induced apoptosis, enhanced cleaved caspase 3 expression, reduced pro-caspase 3 levels and inhibited the protein and mRNA expression of Wnt3a and $\beta$-catenin. However, inhibition of miR-451a demonstrated the opposite effects. In conclusion, the results of the present study revealed that propofol exerted an anti-proliferative and apoptosis-inducing role in ovarian granulosa cells through mediation of miR-451a expression. In addition, the data indicated that miR-451a may be used as an effective therapeutic target for PCOS treatment.

\section{Introduction}

Polycystic ovary syndrome (PCOS) is a universal endocrine disorder, with an incidence of 5-10\% in women of childbearing age globally $(1,2)$. The major clinical symptoms in patients with PCOS are subfertility, amenorrhea, seborrhea and acanthosis $(3,4)$. The etiopathogenesis of PCOS is complicated and its exact mechanism has not been elucidated (5). Therefore, it is critical to understand the mechanisms of PCOS and provide new insight into PCOS therapy.

Propofol is an anesthetic drug frequently-used during clinical practice $(6,7)$. It has been reported that propofol exerts anti-inflammatory and anti-tumor effects on several cancer types, including gastric (8), colorectal (9) and pancreatic (10). Moreover, previous studies have revealed that propofol may suppress cancer cell growth. Gao et al (10) concluded that propofol could inhibit pancreatic cancer progression under hypoxia by ADAM8. Yang et al (11) demonstrated that propofol could suppress lung cancer cell growth and promote cell apoptosis by altering microRNA (miR)-486 expression. However, whether propofol plays similar roles in PCOS remains unclear. Therefore, in the present study, the role of propofol was investigated in ovarian granulosa cell viability and apoptosis and the associated molecular pathways were determined.

Previous studies have suggested that the quantity of growing follicles in PCOS is associated with ovarian granulosa cell proliferation and genetic factors, such as miRNAs (12). miRNAs, with a length of 21 to 23 nucleotides, can impact 
biological functions by binding to the 3'-untranslated region (UTR) of target mRNAs (13). miRNAs participate in the progression of several diseases, including PCOS, and have therefore become promising targets for PCOS treatment. Specific miRNA examples include miR-320 (14) miR-99a (15) and miR-21 (16). miR-451a is located on chromosome 17q11.2. Previous studies indicated that miR-451a was downregulated in various tumors based on the expression of the Ago2 protein, such as papillary thyroid cancer (17), hepatocellular carcinoma (18), non-small cell lung cancer (19) and PCOS (20). Moreover, miR-451a can regulate cell proliferation, apoptosis and metastasis and improve the therapeutic effects of drugs. Based on this available information, the present study explored the role and potential regulatory mechanism of miR-451a in PCOS.

Granulosa cells (GCs), follicular cells surrounding oocytes, ignite a continuous cross-talk between somatic and germ cells (21). GC dysfunction may be involved in various reproductive endocrine disorders including PCOS (22-24). Granulosa cells have been widely used to investigate PCOS in vitro $(24,25)$. And human ovarian granulosa cell-like KGN cells are often used as a model of ovarian granulosa cells to study PCOS in vitro $(25,26)$.

The present study aimed to elucidate the effects of propofol on ovarian granulosa cell proliferation and apoptosis and illustrate the mechanisms associated with this process.

\section{Materials and methods}

Cell culture. Human ovarian granulosa cell-like KGN cells were purchased from ATCC. The cells were maintained in DMEM/F12 medium (Gibco; Thermo Fisher Scientific, Inc.) with 10\% FBS (Gibco; Thermo Fisher Scientific, Inc.), $100 \mathrm{U} / \mathrm{ml}$ penicillinG and streptomycin sulfate (Invitrogen; Thermo Fisher Scientific, Inc.) in a humidified incubator with $5 \% \mathrm{CO}_{2}$ at $37^{\circ} \mathrm{C}$. For drug-toxicity assays, the cells were cultured in 6-well plates and exposed to different concentrations of propofol $(0,1,5$ and $10 \mu \mathrm{g} / \mathrm{ml})$ for $48 \mathrm{~h}(27)$.

Cell transfection and reagents. A total of 50 mimic control (5'-AUCUGA ACGGAUCCUUAUUAAC-3'), $50 \mathrm{miR}-451 \mathrm{a}$ mimic (5'-AAACCGUUACCAUUACUGAGUU-3'), 100 inhibitor control (5'-UUGUACUACAAAAGUACUG-3') or $100 \mathrm{nM}$ miR-451a inhibitor (5'-AACUCAGUAAUGGUA ACGGUUU-3') sequences were obtained from Shanghai GenePharma Co., Ltd. and transfected into KGN cells $\left(5 \times 10^{4}\right.$ cells per well) using Lipofectamine ${ }^{\circledR} 2000$ reagent (Invitrogen; Thermo Fisher Scientific, Inc.) as determined by the manufacturer's instructions. Following transfection, the cells were obtained for subsequent analysis. At $48 \mathrm{~h}$ after cell transfection, subsequent experiments were performed.

Measurement of cell viability. MTT assay was employed to assess the viability of KGN cells. The cells were transfected with mimic control, miR-451a mimic, inhibitor control and miR-451a inhibitor sequences or exposed to propofol. Subsequently, $20 \mu 1$ MTT (Sigma-Aldrich; Merck KGaA) was added into each well and the samples were incubated for $4 \mathrm{~h}$. The culture medium was removed and the cells were dissolved in $150 \mu \mathrm{l}$ DMSO (Sigma-Aldrich; Merck KGaA) in the dark for $10 \mathrm{~min}$. Absorbance was determined at $570 \mathrm{~nm}$ using a microplate reader (Bio-Rad Laboratories, Inc.).

Flow cytometry analysis. KGN cells were stimulated with propofol or transfected with mimic control or miR-451a mimic for $48 \mathrm{~h}$ and the induction of apoptosis was measured by flow cytometry. Subsequently, the cells were washed, centrifuged and harvested and Annexin V-FITC and PI (Annexin-V/PI Apoptosis Detection kit; Beyotime Institute of Biotechnology) were added at room temperature for $15 \mathrm{~min}$ in the dark as described by the manufacturer's protocol. A standard flow cytometer (BD Biosciences) was used to measure the number of apoptotic cells. The data were analyzed using Cell Quest software (version 5.1; BD Biosciences).

Reverse transcription-quantitative $(R T-q) P C R$ analysis. TRIzol $^{\circledR}$ reagent (Invitrogen; Thermo Fisher Scientific, Inc.) was used to extract total RNA from KGN cells following the manufacturer's protocol. Subsequently, cDNA was synthesized from the total RNA using a cDNA Synthesis kit (Takara Bio, Inc.). RT conditions were as follows: $25^{\circ} \mathrm{C}$ for $5 \mathrm{~min}, 42^{\circ} \mathrm{C}$ for $60 \mathrm{~min}$ and $80^{\circ} \mathrm{C}$ for $2 \mathrm{~min}$. RT-qPCR was conducted using an ABI 7500 Real-Time PCR System (Agilent Technologies, Inc.) with SYBR Green Master mix (Takara Bio, Inc.). The samples were initially incubated at $95^{\circ} \mathrm{C}$ for $10 \mathrm{~min}$ to denature the cDNA strand and 37 cycles were performed including denaturation at $95^{\circ} \mathrm{C}$ for $30 \mathrm{sec}$, annealing at $60^{\circ} \mathrm{C}$ for $60 \mathrm{sec}$ and extension at $72^{\circ} \mathrm{C}$ for $15 \mathrm{sec}$. A final extension was conducted at $72^{\circ} \mathrm{C}$ for $10 \mathrm{~min}$. GAPDH was used as the internal reference for mRNA and U6 for miRNA. Primer sequences for PCR were as follows: GAPDH forward, 5'-TTTGGTATC GTGGAAGGACTC-3' and reverse, 5'-GTAGAGGCAGGG ATGATGTTCT-3'; U6 forward, 5'-GCTTCGGCAGCACAT ATACTAAAAT-3' and reverse, 5'-CGCTTCACGAATTTG CGTGTCAT-3'; Wnt3a forward, 5'-GTTCGGGAGGTTTGG G-3' and reverse, 5'-CCAGGAAAGCGGACCAT-3'; $\beta$-catenin forward, 5'-GTTGTACTGCTGGGACCCTT-3' and reverse, 5'-CCCAAGCATTTTCACCAGCG-3'; and miR-451a forward, 5'-ACACTCCAGCTGGGAAACCGTTACCATTACT-3' and reverse, 5'-CTGGTGTCGTGGAGTCGGCAA-3'. The gene expression levels were calculated using the $2^{-\Delta \Delta \mathrm{Cq}}$ method (28).

Western blot analysis. Following treatment with propofol, total protein was isolated from KGN cells using RIPA lysis solution (Beyotime Institute of Biotechnology). The BCA protein quantitative kit (Beijing Solarbio Science \& Technology Co., Ltd.) was employed to measure the protein concentration in accordance with the manufacturer's instructions. Subsequently, the extracted protein samples (40 $\mu \mathrm{g}$ per lane) were loaded on $10 \%$ SDS-PAGE and transferred on PVDF membranes. The membranes were blocked with $5 \%$ skimmed milk at room temperature for $1 \mathrm{~h}$ and incubated with the following primary antibodies: Wnt3a (cat no. ab219412; Abcam), $\beta$-catenin (cat no. ab16051; Abcam), cleaved caspase3 (cat no. ab32042; Abcam), pro-caspase3 (cat no. ab32499; Abcam) and GAPDH (cat no. ab9485; Abcam) at a dilution of 1:1,000 overnight at $4^{\circ} \mathrm{C}$. The following morning, the membranes were incubated with the corresponding secondary antibody (cat no. ab7090; 1:2,000; Abcam) for $1 \mathrm{~h}$ at room temperature. The protein bands 

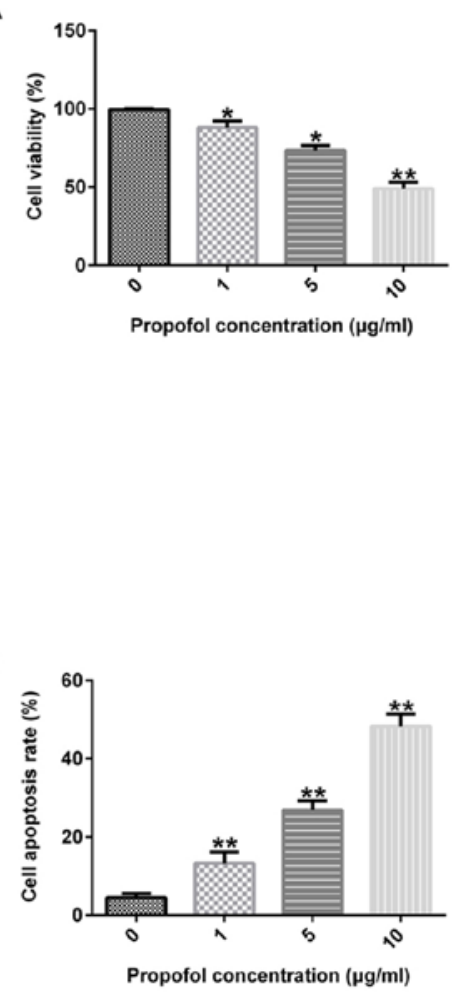

B
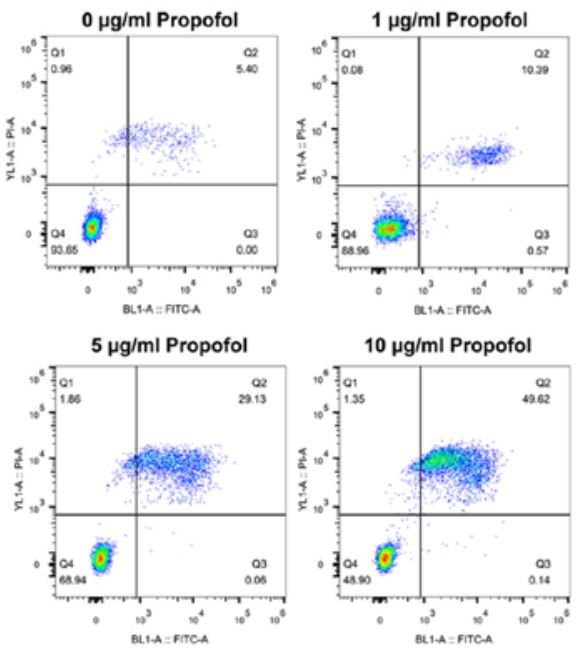

D

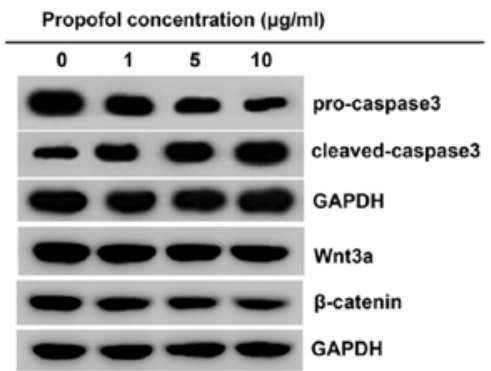

E

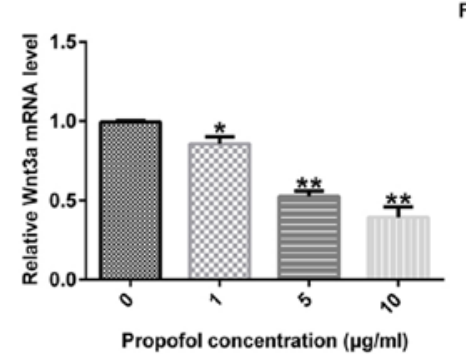

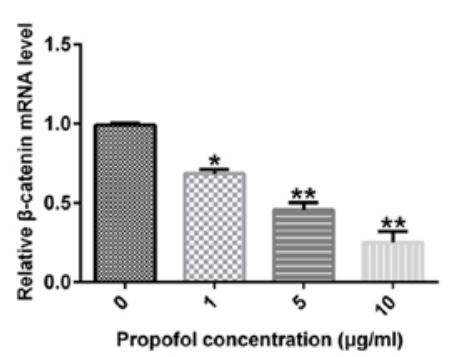

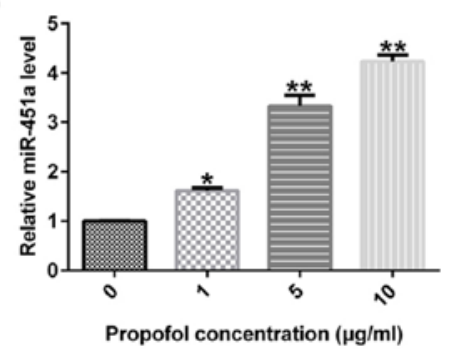

Figure 1. Propofol inhibits KGN cell viability and induces apoptosis. KGN cells were exposed to various concentrations of propofol $(0,1,5$ and $10 \mu \mathrm{g} / \mathrm{ml})$ for $48 \mathrm{~h}$. (A) The cell proliferation of KGN cells was measured using the MTT assay. (B) Flow cytometry assay was carried out to detect the induction of cell apoptosis. (C) Quantification of the rate of apoptosis. (D) Pro-caspase 3, cleaved-caspase 3, Wnt3a and $\beta$-catenin expression levels were determined by western blot analysis. The mRNA expression levels of the apoptosis-associated proteins (E) Wnt3a and (F) $\beta$-catenin were measured by RT-qPCR. (G) The expression levels of miR-451a were determined using RT-qPCR. ${ }^{*} \mathrm{P}<0.05,{ }^{* *} \mathrm{P}<0.01 \mathrm{vs}$. $0 \mu \mathrm{g} / \mathrm{ml}$ propofol treatment group. RT-qPCR, reverse transcription-quantitative PCR.

were exposed using EasyBlot ECL Kit (Shanghai BestBio) and analyzed using Image $\mathbf{J}$ (National Institutes of Health).

Statistical analysis. The measurement data are presented as the mean \pm SD from at least three independent experiments. Statistical analyses were performed using SPSS 19.0 statistical software. One-way ANOVA with Tukey's post-hoc test was used to compare differences among the groups. $\mathrm{P}<0.05$ was considered to indicate a statistically significant difference.

\section{Results}

Propofol inhibits KGN cell proliferation and induced cell apoptosis. In order to investigate the function of propofol in KGN cells, different concentrations of this compound were used to stimulate the cells for $48 \mathrm{~h}$. Subsequently, MTT and flow cytometry analyses were conducted to evaluate cellular growth and apoptosis, respectively. MTT assay revealed that the reduction of KGN cell viability with propofol treatment in comparison to the control was significant (Fig. 1A). In addition, flow cytometry revealed that propofol treatment led to an increase of apoptosis in KGN cells (Fig. 1B and C). Moreover, western blot analysis revealed that propofol markedly increased cleaved-caspase-3 expression and reduced pro-caspase 3 levels in KGN cells compared with levels in untreated cells (Fig. 1D). Furthermore, the influence of propofol on the Wnt/ $\beta$-catenin signaling pathway were investigated and the data suggested that propofol inhibited Wnt3a and $\beta$-catenin protein (Fig. 1D) and mRNA expression levels (Fig. 1E and F) in KGN cells in comparison with a control. These findings 
A

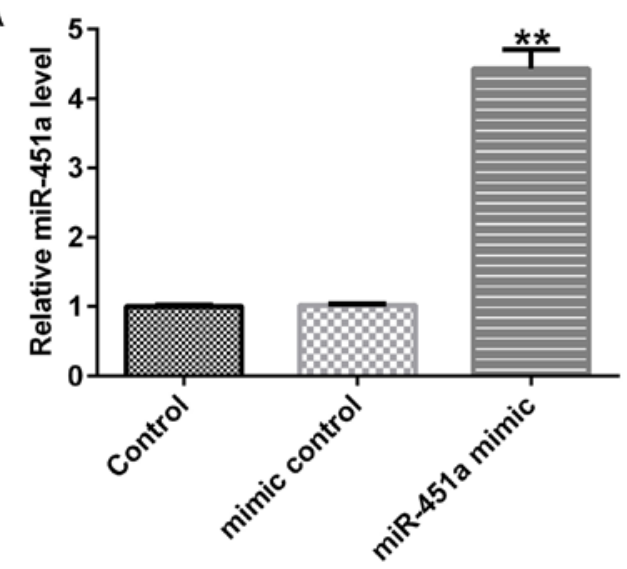

B

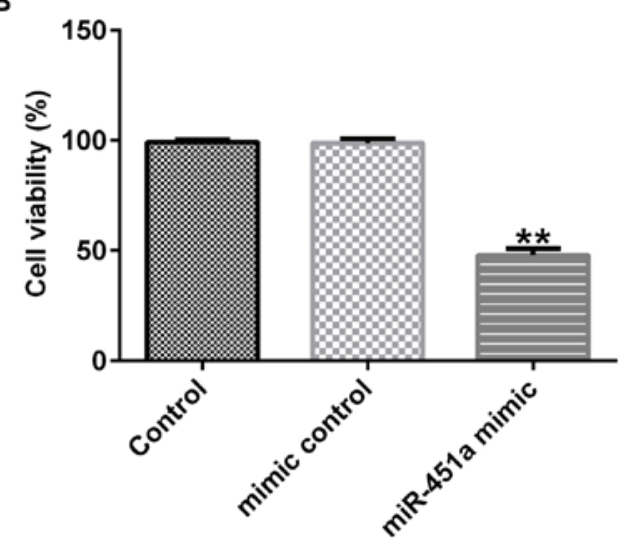

C
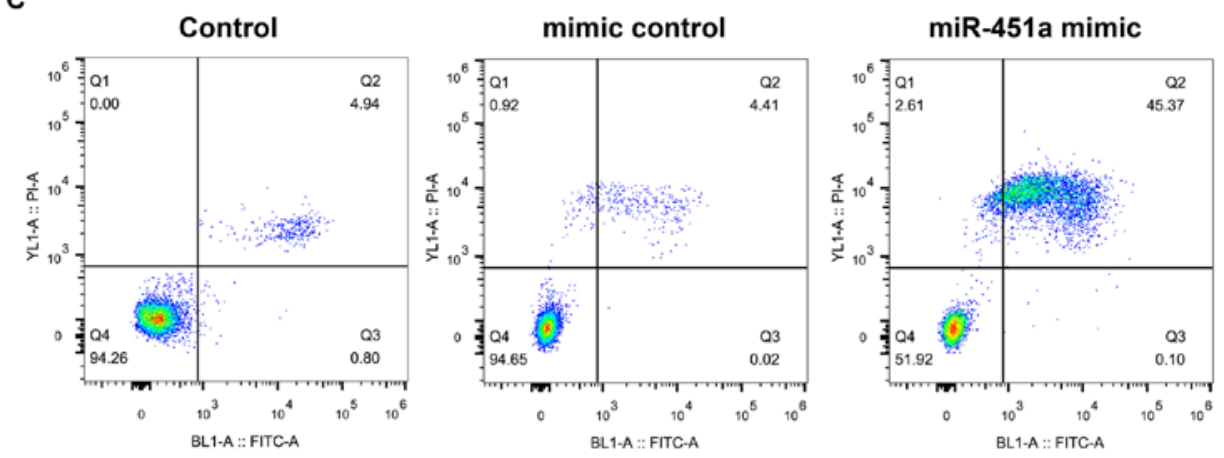

D

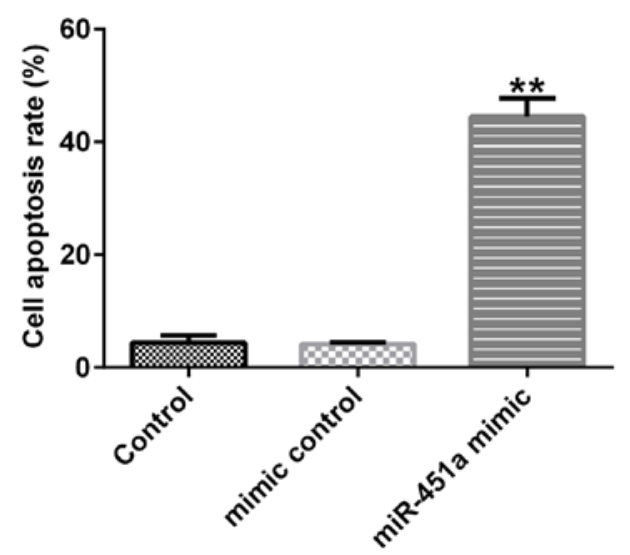

Figure 2. miR-451a mimic suppresses cell proliferation and stimulates apoptosis of KGN cells. Mimic control or miR-451a mimic sequences were transfected into KGN cells for $48 \mathrm{~h}$. Following transfection, (A) the miR-451a levels were detected using RT-qPCR. (B) Cell growth was assessed by the MTT assay. (C) The induction of cell apoptosis was detected using flow cytometry analysis and (D) the apoptotic rate determined. ${ }^{* *} \mathrm{P}<0.01$ vs. mimic control. miR-451a, microRNA-451a; RT-qPCR, reverse transcription-quantitative PCR.

suggested that propofol inhibited cell viability and induced apoptosis of KGN cells and that this was potentially via the $\mathrm{Wnt} / \beta$-catenin signaling pathway. In addition, the data suggested that propofol enhanced miR-451a expression levels in KGN cells in comparison with control (Fig. 1G), suggesting that it may have the potential to suppress PCOS progression.

miR-45la mimic inhibits KGN cell viability and induced cell apoptosis. To examine the effects of miR-451a on KGN cells, mimic control or miR-451a mimic sequences were transfected into KGN cells and the cells were incubated for $48 \mathrm{~h}$. Upregulation of miR-451a promoted miR-451a expression in KGN cells compared with that noted in untreated control and mimic control cells (Fig. 2A). Furthermore, cell proliferation was inhibited (Fig. 2B) and apoptosis was promoted in KGN cells following miR-451a mimic transfection (Fig. 2C and D). These data suggested that miR-451a may participate in the development of PCOS.

miR-45la inhibitor eliminates the effects of propofol on KGN cell growth and on the induction apoptosis. To evaluate the association between miR-451a expression and propofol, inhibition experiments were carried out. KGN cells were transfected with miR-451a inhibitor and inhibitor control sequences for $6 \mathrm{~h}$ using Lipofectamine ${ }^{\circledR} 2000$ reagent. Following transfection, KGN cells were exposed to $10 \mu \mathrm{g} / \mathrm{ml}$ propofol for $48 \mathrm{~h}$. The cells were divided into the following groups: Control, propofol, propofol + inhibitor control and 
A

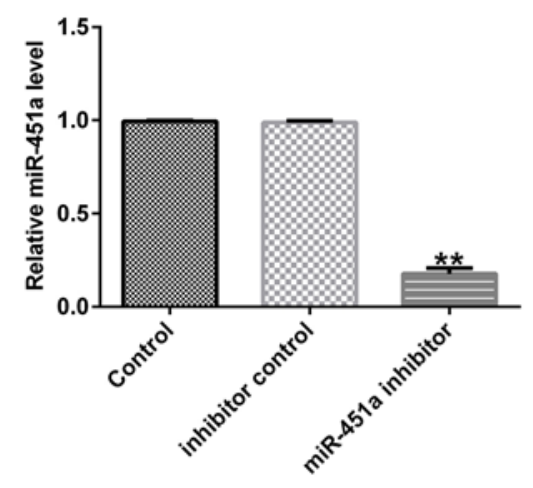

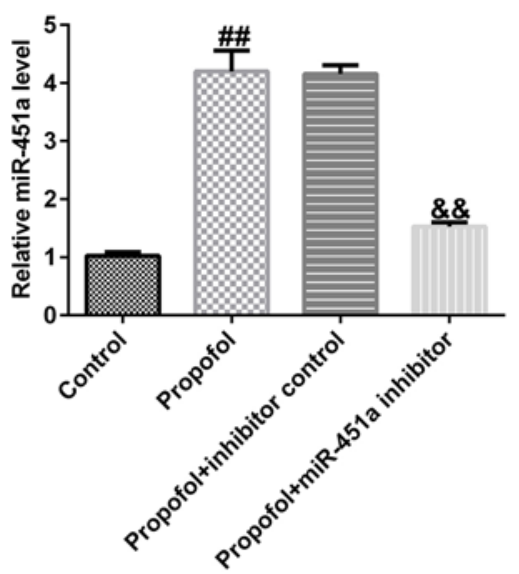

C

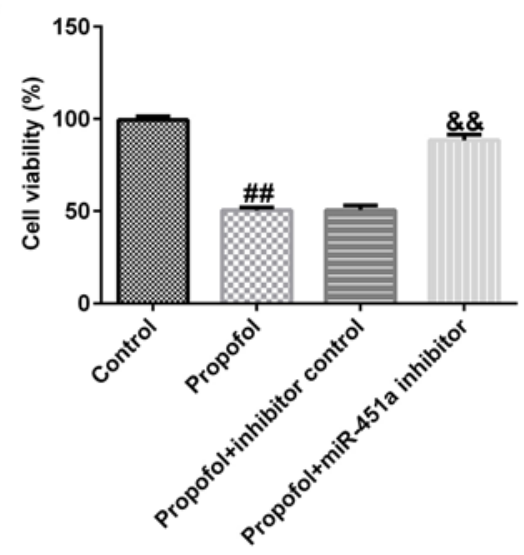

D
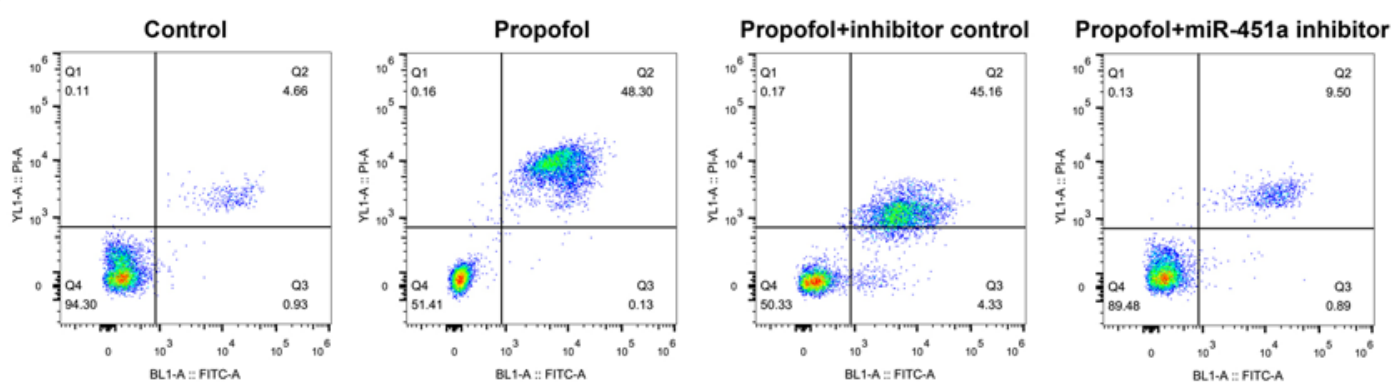

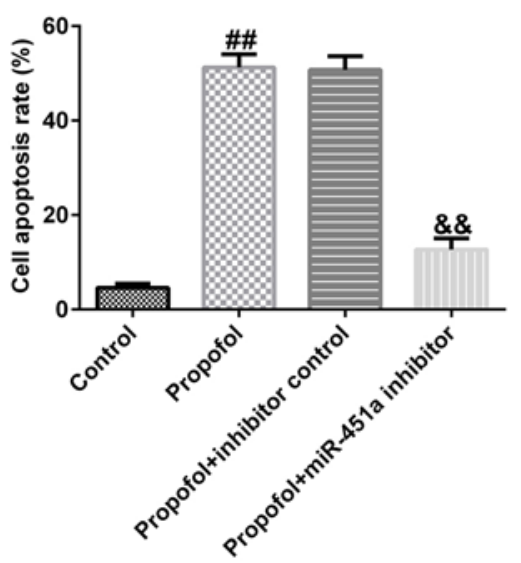

$\mathrm{F}$

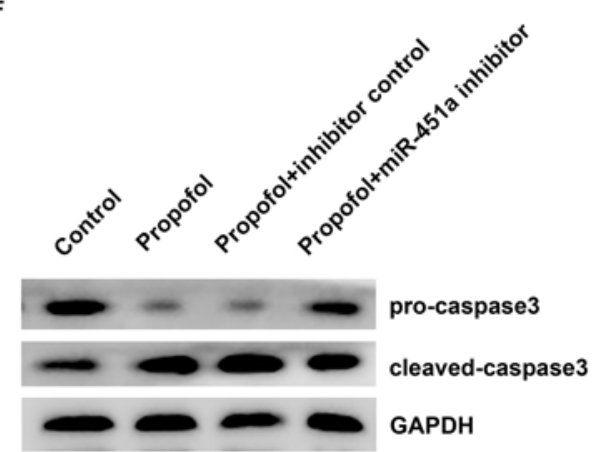

Figure 3. The effects of propofol on KGN cell proliferation and on the induction of apoptosis are reversed by inhibition of miR-451a expression. KGN cells were transfected with miR-451a inhibitor and inhibitor control sequences for $6 \mathrm{~h}$ and treated with $10 \mu \mathrm{g} / \mathrm{ml}$ propofol for $48 \mathrm{~h}$. The cells were divided into the four following groups: Control, propofol, propofol + inhibitor control and propofol + miR-451a inhibitor. (A) RT-qPCR assay was used to determine miR451a levels in transfected KGN cells. (B) Determination of miR-451a levels in transfected cells after propofol treatment. (C) Cell viability after transfection and propofol treatment. (D) Cell apoptosis levels were observed using flow cytometry and (E) the apoptotic rate calculated. (F) The expression levels of cleaved caspase 3 and pro-caspase 3 were determined by western blot analysis in the four KGN cell groups. ${ }^{* *} \mathrm{P}<0.01$ vs. the inhibitor control group; ${ }^{\# \#} \mathrm{P}<0.01$ vs. the control group; ${ }^{\& \&} \mathrm{P}<0.01$ vs. the propofol + inhibitor control group. miR-451a, microRNA-451a; RT-qPCR, reverse transcription-quantitative PCR.

propofol + miR-451a inhibitor. RT-qPCR revealed that miR-451a inhibitor treatment markedly inhibited miR-451a levels in KGN cells compared with those of the control cells (Fig. 3A). However, miR-451a expression levels were significantly increased in propofol-treated KGN cells compared with those of the control cells (Fig. 3B). The opposite effects were noted in the propofol + miR-451a inhibitor group (Fig. 3B). In addition, cell growth, apoptosis and the expression levels of specific apoptotic proteins were assessed using MTT, flow cytometry and western blot assays, respectively. The data revealed that propofol prominently suppressed KGN cell growth (Fig. 3C), promoted apoptosis (Fig. 3D and E), enhanced cleaved caspase 3 levels and reduced pro-caspase 3 expression (Fig. 3F) compared with the corresponding effects noted in control cells. However, these data were reversed following downregulation of miR-451a. The results indicated that miR-451a expression may regulate the effects of propofol in KGN cells.

miR-45la inhibitor eliminates the inhibition effect of propofol on the Wnt/ $\beta$-catenin signaling pathway in $K G N$ cells. To further illustrate the underlying mechanisms of cell apoptosis following treatment of KGN cells with propofol, the expression levels of apoptosis-associated genes and 
A

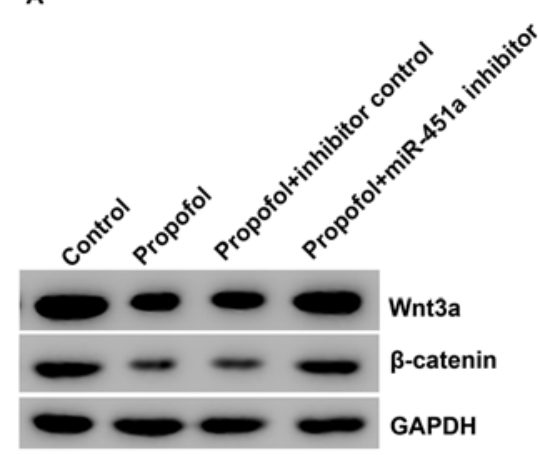

B

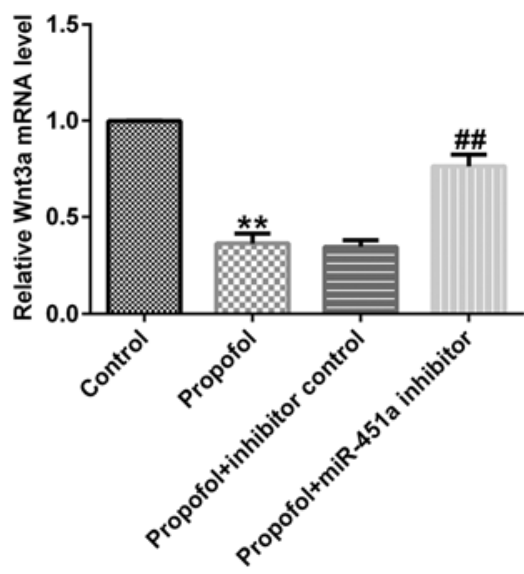

C

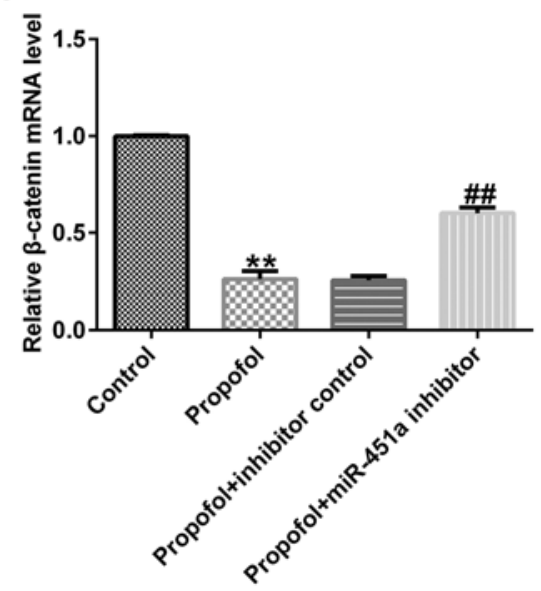

Figure 4. Propofol inactivates the Wnt/ $\beta$-catenin signaling pathway in KGN cells. KGN cells were exposed to $10 \mu \mathrm{g} / \mathrm{ml}$ propofol for $48 \mathrm{~h}$ and subsequently RT-qPCR and western blot assays were used to measure the mRNA and protein expression levels of the genes and proteins involved in this pathway. (A) Wnt3a and $\beta$-catenin protein expression was observed using western blot analysis. The mRNA levels of (B) Wnt3a and (C) $\beta$-catenin were evaluated. ${ }^{* *} \mathrm{P}<0.01$ compared with the control group; ${ }^{\# \#} \mathrm{P}<0.01$ compared with the propofol + inhibitor control group. RT-qPCR, reverse transcription-quantitative PCR.

proteins were determined in these cells using RT-qPCR and western blot assays, respectively. Lower Wnt3a and $\beta$-catenin protein (Fig. 4A) and mRNA levels (Fig. 4B and C) were observed in propofol-stimulated KGN cells than those noted in the control group, suggesting that miR-451a may regulate the protective function of propofol in ovarian granulosa cell proliferation and apoptosis through the $\mathrm{Wnt} / \beta$-catenin pathway.

\section{Discussion}

Propofol is one of the most frequently used anesthetics $(6,7)$. This compound exhibits anti-tumorigenic or anti-phlogotic effects on several cancer cell lines. Yu et al (29) demonstrated that propofol exhibited neuroprotective effects on glutamic acid treated PC12 cells by downregulating miR-19a expression. Moreover, Li et al (9) indicated that propofol inhibited colorectal cancer cell viability and metastasis by regulating the miR-124-3p.1/AKT3 axis. PCOS is a common endocrine disorder with approximate incidence of $10 \%$ in women worldwide (1). The common clinical manifestations of PCOS include hyperandrogenemia, chronic anovulation and sclerocystic ovaries $(2,3)$. However, the therapeutic methods for PCOS patients are limited and its pathogenesis is not fully explored (5).

In the present study, the function of propofol was assessed in ovarian granulosa cells. KGN cells were cultured following treatment with various concentrations of propofol $(0,1,5$, and $10 \mu \mathrm{g} / \mathrm{ml})$ for $48 \mathrm{~h}$. Propofol suppressed cellular growth and promoted cell apoptosis in KGN cells. Cleaved-caspase 3 and pro-caspase 3 are important regulators in cell apoptotic signaling and lead to apoptotic cell death (30). The data presented in the current report are in line with our previous investigations demonstrating that propofol enhanced cleaved-caspase 3 expression and inhibited pro-caspase 3 levels in ovarian granulosa cells $(31,32)$. Accumulating evidence has indicated that the change in the expression levels of Wnt/ $\beta$-catenin signaling proteins may affect the expression levels of proteins involved in various diseases $(33,34)$. The Wnt/ $\beta$-catenin signaling pathway participates in specific cell functions, including cell growth (35), differentiation (36) and apoptosis (37). Therefore, the expression levels of the proteins associated with the Wnt/ $\beta$-catenin pathway were investigated. The results of western blot analysis and RT-qPCR indicated that propofol reduced $\mathrm{Wnt} 3 \mathrm{a}$ and $\beta$-catenin protein and mRNA expression levels. These results revealed that propofol may inhibit cell proliferation and promote cell apoptosis via the $\mathrm{Wnt} / \beta$-catenin signaling pathway, suggesting that it could serve as an appropriate target for PCOS treatment.

miRNAs are small RNAs, which exert key roles by abnormal expression in various human diseases such as cancer (38) and neurodegenerative and cardiovascular disease (39). Previous studies have indicated that miRNAs are associated with several processes involved in tumorigenesis, including cell differentiation, proliferation and apoptosis $(40,41)$. miR-451a has been reported as a noninvasive biomarker in several types of cancer. For example, Liu et al (42) demonstrated that miR-451a inhibited breast cancer cell proliferation and improved tamoxifen sensitivity by regulating macrophage migration inhibitory factor. The results of the present study are in agreement with these findings indicating that miR-451a expression was upregulated in propofol-treated KGN cells compared with that noted in the control cells. This in turn suggested that miR-451a levels may influence the function of propofol in PCOS. However, the expression level of miR-451a in ovarian tissue or ovarian granulosa cells from PCOS patients and normal women was not assessed in the present study, which was a limitation.

In addition, the influence of miR-45la on ovarian granulosa cells was explored. KGN cells were transfected with mimic control or miR-451a mimic for $48 \mathrm{~h}$. The transfection efficiency was assessed by RT-qPCR analysis. The expression of miR-415a was increased in KGN cells compared with that of the control cells. Furthermore, the results from the MTT and flow cytometry assays suggested that miR-451a mimic inhibited KGN cell viability and facilitated apoptosis. Moreover, miR-451a inhibitor and inhibitor control sequences were transfected in KGN cells for $6 \mathrm{~h}$ and miR-451a expression was determined using RT-qPCR. The data demonstrated that miR-451a expression was downregulated in KGN cells following transfection. These results indicated that miR-451a may be involved in the progression of PCOS. 
Several reports have shown that the mechanism of action of propofol is regulated by specific genes during disease progression $(43,44)$. For example, Ren and Zhang (43) suggested that propofol accelerated colorectal cancer cell apoptosis by alleviating the inhibition of lncRNA HOXA11-AS on miRNA let-7i. Sun et al (44) reported that propofol inhibits JEG-3 choriocarcinoma cell proliferation and metastasis by upregulating miR-495. Therefore, inhibition experiments were carried out to evaluate whether miR-451a regulates the effects of propofol. KGN cells were transfected with inhibitor control or miR-451a inhibitor sequences for $6 \mathrm{~h}$. Following transfection, KGN cells were treated with $10 \mu \mathrm{g} / \mathrm{ml}$ propofol for $48 \mathrm{~h}$. The cells were divided into the four following groups: Control, propofol, propofol + inhibitor control and propofol + miR-451a inhibitor. The results demonstrated that propofol increased miR-451a expression, reduced KGN cell growth, promoted apoptosis, enhanced cleaved caspase 3 levels and reduced pro-caspase 3 expression compared to the corresponding effects noted in the control cells. However, these effects were reversed following inhibition of miR-451a. The results revealed that miR-451a expression regulated the functions of propofol in KGN cells.

The interaction of miR-451a and propofol was also examined with regard to the regulation of the $\mathrm{Wnt} / \beta$-catenin pathway. The data suggested that propofol suppressed the mRNA and protein expression levels of $\beta$-catenin and Wnt3a in KGN cells compared with those noted in the control cells. Moreover, the opposite effects were noted in the propofol + miR-451a inhibitor group compared with those of the propofol + inhibitor control group.

In conclusion, the present study provided evidence that propofol exerts anti-proliferative and apoptosis-inducing effects on ovarian granulosa cells by regulating miR-451a expression. This effect contributed partially to the deactivation of the $\mathrm{Wnt} / \beta$-catenin pathway. The findings indicate that propofol may be a promising target for PCOS treatment. However, this study was only a preliminary in vitro study of propofol and miR-451 association. In order to clarify the role of propofol/miR-451a in polycystic ovary syndrome in-depth research is needed. For example, the target genes of mir-451a must be analyzed and rescue experiments are needed to prove the mechanism of miR-451a's effect on KNG cells. Whether propofol/miR-451a directly regulates the Wnt/ $\beta$-catenin pathway in KNG cells requires further in-depth research. Additionally, the role of propofol/miR-451a in polycystic ovary syndrome should be investigated in vivo. Moreover, the expression of miR-451a in ovarian tissue or ovarian granulosa cells from PCOS patients should be determined, and the relationship between miR-451a expression and the clinicopathological parameters of PCOS patients needs to be explored. These studies will be performed in the future.

\section{Acknowledgements}

Not applicable.

\section{Funding}

No funding was received.

\section{Availability of data and materials}

The datasets used and/or analyzed during the current study are available from the corresponding author on reasonable request.

\section{Authors' contributions}

RD and WK contributed to study design, data collection, statistical analysis, data interpretation and manuscript preparation. DW contributed to statistical analysis and data interpretation. LW contributed to statistical analysis and manuscript preparation. RD, WK, DW and LW confirm the authenticity of all the raw data. All authors read and approved the final manuscript.

\section{Ethics approval and consent to participate}

Not applicable.

\section{Patient consent for publication}

Not applicable.

\section{Competing interests}

The authors declare that they have no competing interests.

\section{References}

1. Li X, Zhang T, Li S, Deng Y, Wang L, Tao T, Wang S, Gu Y, $\mathrm{Gu} \mathrm{W}$, Hong J, et al: Correlation between glucose metabolism and serum steroid hormones in patients with polycystic ovary syndrome. Clin Endocrinol (Oxf) 92: 350-357, 2020.

2. Xie L, Zhang D, Ma H, He H, Xia Q, Shen W, Chang H, Deng Y, Wu Q, Cong J, et al: The effect of berberine on reproduction and metabolism in women with polycystic ovary syndrome: A systematic review and meta-analysis of randomized control trials. Evid Based Complement Alternat Med 2019: 7918631, 2019.

3. Berger JJ and Bates GJ: Optimal management of subfertility in polycystic ovary syndrome. Int J Womens Health 6: 613-621, 2014.

4. Dong Z, Huang J, Huang L, Chen X, Yin Q and Yang D: Associations of acanthosis nigricans with metabolic abnormalities in polycystic ovary syndrome women with normal body mass index. J Dermatol 40: 188-192, 2013.

5. Bednarska S and Siejka A: The pathogenesis and treatment of polycystic ovary syndrome: What's new? Adv Clin Exp Med 26: 359-367, 2017.

6. Zhang YF, Li CS, Zhou Y and Lu XH: Effects of propofol on colon cancer metastasis through STAT3/HOTAIR axis by activating WIF-1 and suppressing Wnt pathway. Cancer Med 9: $1842-1854,2020$

7. Li X, Huang L, Zhao Z, Bo L, Kang R, Yang J and Dong Z: The protective effect of the Rho-kinase inhibitor hydroxyfasudil on propofol-induced hippocampal neuron apoptosis in neonatal rats. Int J Clin Exp Pathol 11: 4562-4570, 2018.

8. Ni YJ, Lu J and Zhou HM: Propofol suppresses proliferation, migration and invasion of gastric cancer cells via regulating miR-29/MMP-2 axis. Eur Rev Med Pharmacol Sci 23: 10177, 2019.

9. Li Y, Dong W, Yang H and Xiao G: Propofol suppresses proliferation and metastasis of colorectal cancer cells by regulating miR-124-3p.1/AKT3. Biotechnol Lett 42: 493-504, 2020.

10. Gao Y, Yu X, Zhang F and Dai J: Propofol inhibits pancreatic cancer progress under hypoxia via ADAM8. J Hepatobiliary Pancreat Sci 26: 219-226, 2019.

11. Yang N, Liang Y, Yang P, Yang T and Jiang L: Propofol inhibits lung cancer cell viability and induces cell apoptosis by upregulating microRNA-486 expression. Braz J Med Biol Res 50: e5794, 2017. 
12. Xue Y, Lv J, Xu P, Gu L, Cao J, Xu L, Xue K and Li Q: Identification of microRNAs and genes associated with hyperandrogenism in the follicular fluid of women with polycystic ovary syndrome. J Cell Biochem 119: 3913-3921, 2018.

13. Vicchio TM, Aliquò F, Ruggeri RM, Ragonese M, Giuffrida $G$ Cotta OR, Spagnolo F, Torre ML, Alibrandi A, Asmundo A, et al MicroRNAs expression in pituitary tumors: Differences related to functional status, pathological features, and clinical behavior J Endocrinol Invest 43: 947-958, 2020.

14. Rashad NM, Ateya MA, Saraya YS, Elnagar WM, Helal KF, Lashin ME, Abdelrhman AA, Alil AE and Yousef MS Association of miRNA-320 expression level and its target gene endothelin-1 with the susceptibility and clinical features of polycystic ovary syndrome. J Ovarian Res 12: 39, 2019.

15. Geng Y, Sui C, Xun Y, Lai Q and Jin L: MiRNA-99a can regulate proliferation and apoptosis of human granulosa cells via targeting IGF-1R in polycystic ovary syndrome. J Assist Reprod Genet 36: 211-221, 2019.

16. Jiang L, Li W, Wu M and Cao S: Ciculating miRNA-21 as a biomarker predicts polycystic ovary syndrome (PCOS) in patients. Clin Lab 61: 1009-1015, 2015.

17. Fan $X$ and Zhao Y: miR-451a inhibits cancer growth, epithelial-mesenchymal transition and induces apoptosis in papillary thyroid cancer by targeting PSMB8. J Cell Mol Med 23: 8067-8075, 2019.

18. Wei GY, Hu M, Zhao L and Guo WS: MiR-451a suppresses cell proliferation, metastasis and EMT via targeting YWHAZ in hepatocellular carcinoma. Eur Rev Med Pharmacol Sci 23: 5158-5167, 2019.

19. Shen YY, Cui JY, Yuan J and Wang X: MiR-451a suppressed cell migration and invasion in non-small cell lung cancer through targeting ATF2. Eur Rev Med Pharmacol Sci 22: 5554-5561, 2018.

20. Díaz M, Bassols J, López-Bermejo A, de Zegher F and Ibáñez L: Low circulating levels of miR-451a in girls with polycystic ovary syndrome: Different effects of randomized treatments. J Clin Endocrinol Metab 105: dgz204, 2019.

21. Tatone $\mathrm{C}$ and Amicarelli F: The aging ovary-the poor granulosa cells. Fertil Steril 99: 12-17, 2013.

22. Artimani T, Saidijam M, Aflatoonian R, Ashrafi M, Amiri I, Yavangi M, SoleimaniAsl S, Shabab N, Karimi J and Mehdizadeh M: Downregulation of adiponectin system in granulosa cells and low levels of HMW adiponectin in PCOS. J Assist Reprod Genet 33: 101-110, 2016.

23. Xiong Y, Liu T, Wang S, Chi $\mathrm{H}$, Chen $\mathrm{C}$ and Zheng J: Cyclophosphamide promotes the proliferation inhibition of mouse ovarian granulosa cells and premature ovarian failure by activating the lncRNA-Meg3-p53-p66Shc pathway. Gene 596: $1-8,2017$.

24. Kong L, Wang Q, Jin J, Xiang Z, Chen T, Shen S, Wang H, Gao $Q$ and Wang $Y$ : Insulin resistance enhances the mitogen-activated protein kinase signaling pathway in ovarian granulosa cells. PLoS One 12: e0188029, 2017.

25. Huang $X$, Jin J, Shen S, Xia Y, Xu P, Zou X, Wang H, Yi L, Wang Y and Gao Q: Modulation of expression of 17-Hydroxylase/17,20 lyase (CYP17) and P450 aromatase (CYP19) by inhibition of MEK1 in a human ovarian granulosa-like tumor cell line. Gynecol Endocrinol 32: 201-205, 2016.

26. Han XM, Tian PY and Zhang JL: MicroRNA-486-5p inhibits ovarian granulosa cell proliferation and participates in the development of PCOS via targeting MST4. Eur Rev Med Pharmacol Sci 23: 7217-7223, 2019.

27. Feng S and Sun Y: Protective role of propofol in endometriosis and its mechanism. Exp Ther Med 16: 3646-3650, 2018

28. Livak KJ and Schmittgen TD: Analysis of relative gene expression data using real-time quantitative PCR and the 2(-Delta Delta C(T)) method. Methods 25: 402-408, 2001
29. Yu S, Xin W, Jiang Q and Li A: Propofol exerts neuroprotective functions by down-regulating microRNA-19a in glutamic acid-induced PC12 cells. Biofactors 46: 934-942, 2020.

30. Crowley LC and Waterhouse NJ: Detecting cleaved caspase-3 in apoptotic cells by flow cytometry. Cold Spring Harb Protoc: doi:10.1101/pdb.prot087312.

31. Qu ZJ, Qu ZJ, Zhou HB, Xu CS, Zhang DZ and Wang G: Protective effect of remifentanil on myocardial ischemia-reperfusion injury through Fas apoptosis signaling pathway. Eur Rev Med Pharmacol Sci 23: 5980-5986, 2019.

32. $\mathrm{Wu} \mathrm{H}$, Medeiros LJ and Young KH: Apoptosis signaling and BCL-2 pathways provide opportunities for novel targeted therapeutic strategies in hematologic malignances. Blood Rev 32: $8-28,2018$.

33. Nusse R and Clevers $\mathrm{H}$ : Wnt/ $/$-catenin signaling, disease, and emerging therapeutic modalities. Cell 169: 985-999, 2017.

34. Perugorria MJ, Olaizola P, Labiano I, Esparza-Baquer A, Marzioni M, Marin JJG, Bujanda L and Banales JM: Wnt- $\beta$-catenin signalling in liver development, health and disease. Nat Rev Gastroenterol Hepatol 16: 121-136, 2019.

35. Jiang H, Li Y, Li J, Zhang X, Niu G, Chen S and Yao S: Long noncoding RNA LSINCT5 promotes endometrial carcinoma cell proliferation, cycle, and invasion by promoting the Wnt/ $\beta$-catenin signaling pathway via HMGA2. Ther Adv Med Oncol 11: 1758835919874649, 2019.

36. Chen H, Wang S, Chen L, Chen Y, Wu M, Zhang Y, Yu K, Huang Z, Qin L and Mo D: MicroRNA-344 inhibits 3T3-L1 cell differentiation via targeting GSK $3 \beta$ of $\mathrm{Wnt} / \beta$-catenin signaling pathway. FEBS Lett 588: 429-435, 2014

37. Sun H, Gao Y, Lu K, Zhao G, Li X, Li Z and Chang H: Over-expression of Klotho suppresses liver cancer progression and induces cell apoptosis by negatively regulating wnt/ $\beta$-catenin signaling pathway. World J Surg Oncol 13: 307, 2015.

38. Iacona JR and Lutz CS: miR-146a-5p: Expression, regulation, and functions in cancer. Wiley Interdiscip Rev RNA 10: e1533, 2019.

39. Vishnoi A and Rani S: MiRNA biogenesis and regulation of diseases: An overview. Methods Mol Biol 1509: 1-10, 2017.

40. Zhao M, Sun L, Chen S, Li D, Zhang L, He P, Liu X, Zhang L, Zhang $\mathrm{H}$, Yang D, et al: Borna disease virus infection impacts microRNAs associated with nervous system development, cell differentiation, proliferation and apoptosis in the hippocampi of neonatal rats. Mol Med Rep 12: 3697-3703, 2015.

41. Shen S, Luo X, Gao K, Sun Y, Yao D and Zhu L: Identification and integrative analysis of microRNAs and mRNAs involved in proliferation and invasion of pressuretreated human liver cancer cell lines. Mol Med Rep 20: 375-387, 2019.

42. Liu Z, Miao T, Feng T, Jiang Z, Li M, Zhou L and Li H: MiR-451a inhibited cell proliferation and enhanced tamoxifen sensitive in breast cancer via macrophage migration inhibitory factor. Biomed Res Int 2015: 207684, 2015.

43. Ren YL and Zhang W: Propofol promotes apoptosis of colorectal cancer cells via alleviating the suppression of lncRNA HOXA11-AS on miRNA let-7i. Biochem Cell Biol 98: 90-98, 2019.

44. Sun H, Wang Y and Zhang W: Propofol inhibits proliferation and metastasis by up-regulation of miR-495 in JEG-3 choriocarcinoma cells. Artif Cells Nanomed Biotechnol 47: 1738-1745, 2019.

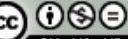

This work is licensed under a Creative Commons Attribution-NonCommercial-NoDerivatives 4.0 International (CC BY-NC-ND 4.0) License. 